\title{
Présentations affichées au 4ème Workshop International sur l'Epididyme.
}

\author{
Chatel Guyon, France \\ du 4 décembre 2006 au 7 décembre 2006
}

\begin{abstract}
Réunion satellite du 4eme Congrès Européen d'Andrologie ECA 2006
\end{abstract}
(Toulouse, du 7 au 10 décembre 2006)

\author{
Organisateurs : Prof. Joël R. Drevet et Dr. Trevor G. Cooper
}

Chatel-Guyon (Puy de Dôme, France) a accueilli en décembre dernier la quatrième édition du Workshop International sur l'Epididyme (EPID-IV). Cette manifestation est à l'épididyme ce que le "Testis Workshop " est à la gonade mâle. Elle a vu le jour à la fin des années 1980 sous l'impulsion de quelques personnalités scientifiques internationales impliquées dans des recherches sur l'épididyme qui ont suggéré que l'épididyme ne devait pas rester le parent pauvre de la gonade et qu'un forum d'échange et de discussion serait bienvenu afin de stimuler le domaine de recherche.

C'est ainsi que le premier " Epididymis Workshop " s'est tenu en 1992 à Hong-Kong (Organisateur : Dr. Patrick Wong) et a été suivi en 1998 par une réunion en Australie (Epididymis II : The Boden Conference on the Epididymis, Newcastle, NSW. Organisateur : Dr. Russel Jones). Epididymis III s'est tenu en 2002 à Charlottesville (Virginie, USA. Organisateurs Drs. Terry T. Turner et Barry Hinton) et la périodicité d'une réunion tous les 4 ans a été définitivement adoptée. Ces réunions rassemblent pratiquement la totalité des chercheurs à l'international travaillant sur l'épididyme des mammifères. Cela concerne un effectif assez discret: aux alentours d'une centaine de chercheurs de par le monde. Le caractère international de la manifestation et sa périodicité en font un événement majeur dans le champ d'investigation concerné.

Dans sa quatrième édition, EPID-IV a rassemblé plus de 95 personnes de 14 nationalités différentes. La manifestation a reçu le support de l'industrie pharmaceutique (Wyeth-Ayerst Inc. Mineapolis, USA; Schering $A G$, Berlin, Germany) de différentes institutions internationales (The Lalor Foundation, Boston, USA; CONRAD, New-York, USA) et nationales (CNRS, INRA, Ministère de la recherche et de l'enseignement supérieur, Université Blaise Pascal-Clermont2) et de sociétés ou de fédération de sociétés savantes internationales (International Society of Andrology, European Academy of Andrology, British Andrology Society) et nationale (SALF). Tous ces " sponsors " sont vivement remerciés. Sans leurs soutiens, cette manifestation n'aurait pu avoir lieu.

Pourquoi s'intéresser à l'épididyme, cet obscur tuyau par lequel transitent et sont stockés les spermatozoïdes entre deux éjaculations?

II est vite apparu que si les spermatozoïdes qui quittent le testicule, siège de la spermatogenèse, semblent être structurellement finis, ils n'en sont pas moins fonctionnellement immatures. En effet, à la sortie de la 
gonade mâle les spermatozoïdes ne sont pas fécondants, ce qui peut se traduire par le fait qu'ils ne peuvent pas reconnaître et pénétrer un ovule, ni ne sont capables de se servir de leur flagelle et d'être motiles. Ces aptitudes sont acquises progressivement au cours de leur descente dans le canal épididymaire et résultent essentiellement d'une série complexe de modifications séquentielles des gamètes dans le micro-environnement du fluide épididymaire.

Ces modifications sont le seul fait des activités de synthèse et de sécrétion/réabsorption de l'épithélium épididymaire car les gamètes sont à ce stade transcriptionnellement silencieux. Le micro-environnement luminal dans lequel baignent les spermatozoïdes change en tout point de l'organe reflétant le caractère séquentiel et dynamique de cette maturation post-testiculaire des gamètes mâles. C'est aussi par le biais de ces activités de synthèse de l'épithélium épididymaire qu'est assurée la protection/survie des gamètes mâles pendant leur maturation fonctionnelle et durant les phases de stockage dans la queue de l'épididyme entre deux éjaculations.

Comprendre la physiologie de cet organe se révèle être particulièrement important tant sur le plan fondamental, qu'appliqué. En effet, un nombre non négligeable d'infertilités chez l'homme n'est pas le fait d'une spermatogenèse anormale mais plutôt d'une maturation anormale des gamètes ou d'une fragilité accrue. Connaître les aspects essentiels de la maturation épididymaire des spermatozoïdes permettrait sans doute de comprendre certaines de ces infertilités. Par ailleurs, dans un tout autre aspect, les activités de l'épithélium épididymaire sont autant de voies possibles d'interférence avec la fertilité qui pourraient permettre le développement de nouvelles stratégies contraceptives post-testiculaires et non-hormonales. L'industrie pharmaceutique s'est récemment intéressée à l'épididyme dans cette optique.

Dans cette quatrième édition du Workshop sur l'épididyme, 34 présentations ont été affichées par des spécialistes de divers aspects de la physiologie de l'épididyme. Les résumés de ces présentations affichées ont été traduits pour " Andrologie " et sont présentés ci-après. Le traducteur a respecté strictement les " abstracts " des auteurs, et convie les lecteurs à demander tous les détails qu'ils souhaiteraient avoir aux auteurs.

Professeur Joël R. Drevet 